\title{
Effects of a Digital Self-care Intervention in Adults with COPD: A Pilot Study
}

(C) The Author(s) 2019

Article reuse guidelines:

sagepub.com/journals-permissions DOI: $10.1177 / 0193945919892282$

journals.sagepub.com/home/wjn

(S)AGE

\author{
Andrew Bugajski ' (D), Susan K. Frazier², Lakeshia Cousin', \\ Kaitlyn Rechenberg', Jaelyn Brown', Alexander J. Lengerich', \\ Debra K. Moser², and Terry A. Lennie ${ }^{2}$
}

\begin{abstract}
Self-care improves quality of life, and reduces exacerbations and mortality risk in people with chronic obstructive pulmonary disease (COPD). The purpose of this report is to describe the development and testing of the feasibility and acceptability of a digital, educational self-care intervention designed to improve self-care ability, adherence, knowledge, somatic symptoms, anxiety, and depressive symptoms.

This pilot study was a quasi-experimental, repeated measures design. Self-care behaviors were identified, and educational modules were developed and built into a web-based platform. Twenty participants were enrolled to determine feasibility, acceptability, and preliminary efficacy.

The intervention was deemed acceptable and feasible as evidenced by $>95 \%$ completion rates and high degree of participation. Overall, the intervention was well received and is feasible for future home-based interventions in those with COPD. Results included significant improvements in adherence and knowledge, and significant associations between self-care ability, adherence, and depressive symptoms.
\end{abstract}

\section{Keywords}

adherence, COPD, eHealth, self-care, self-management

Chronic obstructive pulmonary disease (COPD) is predicted to be the third leading cause of death worldwide by 2030 (World Health Organization, 2017). COPD is usually a progressive and irreversible disease characterized by persistent airflow limitation and respiratory symptoms (Global Strategy for the Diagnosis, Management and Prevention of COPD, 2019). Approximately $99 \%$ of patients with COPD reported daily symptoms like dyspnea and fatigue, and up to $80 \%$ of patients with COPD describe feeling anxious and/or depressed (Cannon et al., 2016; Yohannes \& Alexopoulos, 2014). Due to the high symptom burden and frequently progressive nature of COPD, patients with COPD are often required to perform complicated and demanding self-care tasks at home. Self-care is the process by which individuals with chronic disease attain optimal health through learned, intentional health promoting actions; these activities must occur during both healthy and exacerbated states (Riegel, Jaarsma, Lee \& Strömberg, 2018). Recently, an international consensus group of COPD experts described self-care/management interventions as "structured, personalized and often multi-component, with goals of motivating, engaging and supporting the patients to positively adapt their health behavior(s) and develop skills to better manage their disease" - previous interventions falling under this definition have led to improvements in health-related quality of life (HRQoL), decreased risk for allcause hospitalization, reduced anxiety and depressive symptoms in patients with COPD (Effing et al., 2016; Jolly et al., 2016; Zwerink et al., 2014).

\section{Self-Care}

According to the Middle Range Theory of Self-care of Chronic Illness, self-care comprises three related concepts: maintenance, monitoring, and management (Riegel, Jaarsma, $\&$ Strömberg, 2012). Self-care is herein used instead of selfmanagement as it is more encompassing. Each concept contains progressively more complex self-care behaviors that patients with chronic conditions are expected to master over time. Behaviors inherent in each self-care concept can be

'College of Nursing, University of South Florida, Tampa, FL, USA
${ }^{2}$ College of Nursing, University of Kentucky, Lexington, KY, USA

Corresponding Author:

Andrew Bugajski, College of Nursing, University of South Florida, I290 I

Bruce B. Downs Blvd., Tampa, FL 336I2, USA.

Email: abugajski@health.usf.edu 
improved and mastered through the use of targeted educational interventions - this practice is prevalent in studies of other chronic disease like heart failure. However, COPD self-care lacks evidence as to which specific self-care behaviors clinicians should target when educating patients with COPD, as elaborated in two recent Cochrane Reviews on this matter (Lenferink et al., 2017; Zwerink et al., 2014).

Two recent Cochrane reviews including 22 and 29 studies, respectively, examined the efficacy of self-management interventions on outcomes such as HRQoL, hospitalizations, exacerbations, functional capacity, and mortality. In summary, those interventions that included comprehensive selfmanagement programs were found to increase HRQoL, reduce the risk for respiratory related admissions, but did not affect outcomes such as mortality, exacerbation frequency, or functional capacity (Lenferink et al., 2017; Zwerink et al., 2014). Authors of these reviews cited several shortcomings, primarily related to the robustness of included interventions, prohibiting the author's ability to pinpoint which behaviors were effective and at which dose elicited the greatest effect.

Contrarily, researchers have parsed some of these comprehensive interventions into smaller, more manageable programs centered on pulmonary rehabilitation (PR) programs and principles. PR and interventions paired with PR have covered many educational topics pertinent to those with COPD, ranging from exercise to mental well-being, symptom control, and exacerbation planning (Nguyen, Gill, Wolpin, Steele, \& Benditt, 2009; Nguyen, Harrington, Liu, Lee, \& Gould, 2015; Wouters et al., 2018). These interventions have been shown to reduce symptom distress, improve self-care behavior performance, and improve HRQoL. However, one of the key limitations of using PR literature to support self-management interventions relates to the confounding effect of the PR itself. PR is often highly structured and takes place over weeks to months, and in some cases years; this inherently contains formal structured exercise and education, which may not reflect the reality of many patients with COPD.

To date, it is currently unknown how COPD patients interact with educational self-care interventions, and until recently with the advent of digitally delivered interventions, has been difficult to track their engagement and activity. Digitally delivered interventions have numerous benefits compared to traditional paper-based formats: (a) information can be rapidly communicated to patients and providers, (b) detailed usability statistics and intervention interactions can be generated/recorded passively, (c) there is less physical burden compared to traditional paper-format, and (d) the intervention can quickly be adapted and updated over time (Noar \& Harrington, 2012).

\section{Purpose}

Thus, the purpose of this pilot study was to (a) develop an educational self-care intervention using evidence-based selfcare behaviors and (b) determine feasibility and acceptability in a pilot test of the intervention. Our secondary purpose was to collect data that indicated positive and or negative effects from the intervention on the following outcomes: perceived self-care ability, self-care adherence, self-care information needs (knowledge), somatic symptoms, anxiety, and depressive symptoms in adult patients with stable COPD $(\mathrm{N}=20)$.

\section{Methods}

\section{Intervention Development}

We purposively searched primary and secondary sources that reflected the most up-to date self-care research in COPD (Jolly et al., 2016; Lenferink et al., 2017; Zwerink et al., 2014), as well as current clinical guidelines from pulmonary organizations that have a global impact (such as the Global Initiative for Obstructive Lung Disease [GOLD], American Thoracic Society, and COPD Foundation) to identify evidence-based self-care behaviors that could be incorporated into an educational intervention. In total, we identified 83 self-care behaviors, similar to the results produced by Clari et al. (2017). The identified behaviors were analyzed for content and/or theme (within the context of the intervention for some behaviors). Content and/or themes were then synthesized into seven self-care categories as agreed upon by the research team: (a) nutrition and diet, (b) physical activity and exercise, (c) medications, (d) breathing control, (e) mental health, (f) environment, and (g) exacerbation planning.

Each of the 83 behaviors were then labeled to reflect the Riegel et al (2012) self-care theory, including low-mastery behaviors (maintenance), intermediate mastery behaviors (monitoring), or high-mastery behaviors (management; Riegel et al., 2012) - these behaviors were labeled for investigator knowledge, but were not presented as such in the intervention itself. Given our aim to build an evidence-based intervention, the five most evidence-based behaviors from our literature review, in combination with the works from Clari et al. (2017), were chosen for each self-care behavior category, resulting in 35 total behaviors incorporated into this intervention. For each of the seven self-care behavior categories, there were at least one behavior from each mastery level (maintenance, monitoring, and management) to account for the varying self-care ability levels present at baseline. Additionally, behaviors were chosen based upon previous evidence to support its efficacy and/or its recommendation from medical societies. A summary of topics chosen for each self-care category can be seen in Table 1 .

We acknowledge that the PR literature has many studies pertaining to education in COPD. However, in the conceptualization of our intervention, we analyzed the PR literature and deemed that inherent in PR are many latent and confounding effects on patient outcomes, due to the regimented nature of PR, as well as the heavy emphasis on exercise and physical activity. Taking these principles into account, we looked at the PR literature not to necessarily base our 
Table I. Description of the Seven Educational Components Included in the Intervention.

\begin{tabular}{|c|c|}
\hline $\begin{array}{l}\text { Self-Care } \\
\text { Component }\end{array}$ & Content Covered \\
\hline Nutrition/Diet & $\begin{array}{l}\text { Caloric intake and weight management } \\
\text { Appropriated of macronutrients } \\
\text { Meal timing and portions } \\
\text { Water consumption } \\
\text { Vitamins, minerals, and dietary supplements }\end{array}$ \\
\hline $\begin{array}{l}\text { Physical } \\
\text { Activity }\end{array}$ & $\begin{array}{l}\text { Physical activity promotion (ways to improve } \\
\text { light physical activity not normally considered } \\
\text { "exercise") } \\
\text { Recommended exercises for people with } \\
\text { COPD } \\
\text { Amount of exercise per day/week } \\
\text { Breathing control while exercising } \\
\text { Developing prolonged exercise habits (routine } \\
\text { reinforcement) }\end{array}$ \\
\hline Medications & $\begin{array}{l}\text { Compiling an accurate list of medications } \\
\text { Timing and dosing of daily medications } \\
\text { (knowledge and behavior practice) } \\
\text { Non-pharmacologic ways to manage acute } \\
\text { symptom onset } \\
\text { Establishing the most common side effects of } \\
\text { different medications } \\
\text { Establishing an action plan for when symptoms } \\
\text { need acute treatment }\end{array}$ \\
\hline $\begin{array}{l}\text { Breathing } \\
\text { Control }\end{array}$ & $\begin{array}{l}\text { Pursed lip breathing } \\
\text { Diaphragmatic/belly breathing } \\
\text { Utilizing rest to prevent dyspnea } \\
\text { Postural positioning for optimal breathing } \\
\text { Using relaxation techniques to combat the } \\
\text { anxiety-dyspnea cycle }\end{array}$ \\
\hline Mental Health & $\begin{array}{l}\text { Identification of life stressors } \\
\text { Developing a plan to combat common life } \\
\text { stressors } \\
\text { Utilization of breathing techniques to reduce } \\
\text { anxiety/dyspnea } \\
\text { Non-pharmacologic ways to cope with anxiety } \\
\text { meditation, mindfulness, muscle relaxation, } \\
\text { biofeedback, and distraction therapy } \\
\text { Identifying depression and depressive } \\
\text { symptoms }\end{array}$ \\
\hline $\begin{array}{l}\text { Environmental } \\
\text { Modification }\end{array}$ & $\begin{array}{l}\text { Smoking cessation and avoiding lung irritants } \\
\text { Involvement of caregiver/significant other/ } \\
\text { friend/family with medical care } \\
\text { Promoting social interaction } \\
\text { Energy conservation (spacing activities) } \\
\text { Modifying living arrangements }\end{array}$ \\
\hline $\begin{array}{l}\text { Exacerbation } \\
\text { Planning }\end{array}$ & $\begin{array}{l}\text { Developing an action plan } \\
\text { Establishing symptom norms and knowing } \\
\text { when to seek help } \\
\text { Identifying where is most appropriate to seek } \\
\text { medical treatment } \\
\text { Identifying early warning signs of worsening } \\
\text { symptoms } \\
\text { Medication uses when symptoms become } \\
\text { worse }\end{array}$ \\
\hline
\end{tabular}

Note. All materials were delivered via print, pictures, and/or interactive videos. intervention off of previously successful interventions, but rather to examine if certain educational constructs seemed beneficial and translatable to a self-guided intervention such as ours. There were numerous educational concepts extracted from PR literature and included in our intervention.

Incorporation of behaviors into a digital intervention. We then incorporated the five most evidence-based behaviors identified in our literature review into a digital intervention hosted on a website. The website was partitioned into two main sections: (a) the intervention and (b) data collection tools. The intervention was separated into seven distinct modules which reflect the seven synthesized self-care categories identified in our literature search (nutrition and diet, physical activity and exercise, medications, breathing control, mental health, environment, and exacerbation planning). For each of the 35 total behaviors chosen to be incorporated into the intervention, we developed writings, pictures, and/or videos to educate patients on how to preform each behavior, or incorporate the behavior into their activities of daily living.

One of the included behaviors was proper inhaler technique, in which we provided written directions, pictures of proper technique, and videos demonstrating the appropriate behavior. Another example was about environment modification, specifically speaking to altering their living arrangements to conserve energy. This included a list of things they could do, such as using extendable-reach grips to access things on higher shelves, trying to make as many tasks at waist to sternum level (e.g., making coffee and folding laundry), and how to plan their day so that they space out energy intensive activities throughout the course of their day instead of completing them all at once. There were written, visual, and multimedia components built to teach the participants these concepts.

The modules were designed so that introductory teachings encompassed low-level self-care behaviors and the later teachings encompassed high-level self-care teachings. Each module was designed to be open and self-guided, so that participants could access the material in which they believed needed the greatest amount of education. The website was optimized to be viewed and used on a tablet or mobile device. For this pilot study, we provided the participants with a tablet-computer (Amazon Fire Tablet, 8 gigabytes, 7 th generation).

\section{Design}

To study the variables in this report, we used a repeated-measures, quasi-experimental design for this pilot study-the total duration of the study was 3 weeks (21 consecutive days). Participants were assessed at baseline, after the first week (time 1), after the second week (time 2), and after the third week (time 3). Efficacy of the digital intervention was examined by comparing data collected at baseline to data from times 1, 2, and 3. Measures of perceived self-care ability, 
anxiety, and depression, were measured at baseline and at times 1, 2, and 3. Perceived self-care adherence and self-care information needs (knowledge) were measured at baseline and at time 3. As stated previously, this pilot study was intended to test processes; thus sensitivity to change was not prioritized in our measures. We observed which topics the participants accessed the most, and thus which behaviors were likely to contribute to any observed outcomes. Given the exploratory nature of this pilot study, however, we only expected to observe changes in topics that have historically been shown to change over a short duration (knowledge, adherence, and anxiety).

\section{Sample and Setting}

Patients were recruited using a purposive, non-probability sampling method. Patients aged 40 years to 70 years old who were present for routine follow up visits for primary or secondary diagnosis of COPD at a pulmonary clinic in the United States were screened for eligibility. Patients were candidates for inclusion if they (a) had a confirmed diagnosis of COPD verified by spirometry demonstrating moderate to severe disease according to GOLD criteria (forced expiratory volume in one second $\left[\mathrm{FEV}_{1}\right] /$ forced vital capacity $[\mathrm{FVC}]<70 \%$ and $\mathrm{FEV}_{1}<80 \%$ ); (b) had stable disease state defined by absence of an acute exacerbation of COPD (sudden worsening of respiratory symptom requiring medical intervention; self-report and verified by medical records) in last 3 months; (c) had access to home Wi-Fi/internet; (d) could read, write, and speak English; and (e) had a cell-phone with text messaging capabilities. Patients were excluded if they had (a) presence of symptomatic cardiovascular diseases or severe systemic diseases (end-stage liver or renal disease, systemic lupus erythematosus, or malignancy); (b) impaired eyesight prohibiting accurate visualization of tablet font as evidenced by failure to correctly read the tablet home screen application list; (c) obvious cognitive impairment (MiniCog scores $<3$ ) (Borson, Scanlan, Chen, \& Ganguli, 2003); or (d) low health literacy as evidenced by a score of more than four incorrect responses on the Newest Vital Sign instrument (Weiss et al., 2005).

Due to the exploratory nature of this pilot study, a sample of 20 participants, with no control group, were recruited. Given the primary and secondary purposes of this pilot study was to observe the feasibility and acceptability of the intervention while collecting data that indicated positive and or negative effects from the intervention, 20 was deemed sufficient to fulfil our purposes.

\section{Measures}

Clinical and sociodemographic variables. Sociodemographic variables included age, sex, ethnicity, highest education level, marital status, employment status, and living situation. Clinical data included height, weight, body mass index
(BMI), spirometry measures (forced expiratory volume/second $\left[\mathrm{FEV}_{1}\right]$, forced vital capacity $[\mathrm{FVC}], \mathrm{FEV} / \mathrm{FVC}$, reference $\mathrm{FEV}_{1}$, reference $\mathrm{FVC}$ and reference $\mathrm{FEV}_{1} / \mathrm{FVC}$ ), GOLD stage (indicator of severity of disease), number of exacerbations in past year, smoking status (packs per day and pack years), and current prescribed medications. These data were obtained from medical record review and patient interview.

Perceived self-care ability. Self-care ability was defined as "the core behavioral and cognitive abilities which presumably contribute to sustainable well-being" (Cramm, Strating, de Vreede, Steverink, \& Nieboer, 2012). Self-care ability was measured with the Self-Management Ability Scale-Short (SMAS-S; Cramm et al., 2012). It is an 18-item, self-report questionnaire that has been used in patients with COPD and other chronic illnesses (Cramm \& Nieboer, 2013). The SMAS-S total score assesses self-management ability with items in six sub-scales that include taking initiative, investment behavior, variety, multi-functionality, self-efficacy, and positive frame of mind. Subscales vary between a 5-point and a 6-point Likert scale, where higher scores indicate better self-care ability. Each sub-scale can be used independently, or all 18 questions can be used as a total composite score; scores can range from 18 to 102, and there is no established cutoff for this scale. The six sub-scales had satisfactory internal consistency with Cronbach's $\alpha$ ranging from 0.69 to 0.77 . For this pilot study, we only used the composite score as our indicator of self-care ability.

Self-care adherence. Self-care adherence was defined as the extent to which an individual follows the self-care recommendation/prescription (Sherbourne, Hays, Ordway, DiMatteo, \& Kravitz, 1992). Self-care adherence was measured with a modified version of the Medical Outcome Study Specific Adherence Scale (MOS-SAS; Sherbourne et al., 1992). This modified MOS-SAS was built with the investigative team (using the principles of face validity) as an eight-item instrument that assesses adherence for each of the seven self-care domains included in this study. The MOS-SAS evaluates, "How often have you done each of the following in the past week?"; each answer is measured on a 6-point Likert scale ranging from "none of the time" to "all of the time." Specific question topics included were exercising regularly, taking medications as prescribed, using relaxation techniques, stopping or cut down on smoking, following a low carbohydrate diet, cutting down on stress, and comparing symptoms to previous day(s) symptoms. Each response is weighted to achieve a range of possible total score of $0-100$; all items are added then averaged. There are no established cut points for this scale. Internal consistency for the MOS-SAS ranged from 0.50 to 0.78 in similar chronic disease populations who require sustained, lifelong treatment (diabetes, hypertension, and heart failure; Sherbourne et al., 1992). 
Self-care information needs (knowledge). Self-care information needs were defined as the content required by an individual so they can perform self-care (Hyland, Jones, \& Hanney, 2006). Self-care information needs were measured with the Lung Information Needs Questionnaire (LINQ; Hyland et al., 2006). The LINQ is a 16-item questionnaire developed to assess self-care information needs and COPD knowledge in patients with COPD. Six domains comprise the LINQ; these include disease knowledge, medicines, self-management, smoking, diet, and exercise. Item scores for each domain are added to achieve a range of total scores from 0 to 25 , with higher scores indicating higher information needs. There is no set cutoff for adequacy of informational needs. LINQ total score has satisfactory internal consistency with a Cronbach's $\alpha$ of 0.62 (Hyland et al., 2006).

Somatic symptoms. Somatic symptoms were defined as the participant's evaluation of the degree of intensity and change over 24 hours in disease-related symptoms of: distress due to cough, chest tightness, distress due to mucous, dyspnea with normal activity, dyspnea at rest, and fatigue. A modified version of the Daily Symptom Scale (DSS) (Webel, Frazier, Moser, \& Lennie, 2007) was used to measure symptom severity and variability. The 6 -item, modified DDS prompted the participants to rate the severity of their symptoms daily on a 100-point visual analog scale (VAS) where 0 was absence of a symptom and 100 was the most distressful the symptom could be (Webel et al., 2007). The DDS has been shown to be reliable in patients with similar chronic diseases such as cardiovascular disease and heart failure (Webel et al., 2007). Face validity of the modified DDS was confirmed by two pulmonary care experts. Reliability and validity of a computerized VAS has been shown to be low to moderately reliable with test-retest reliability coefficient of 0.56 and demonstrated strong convergent validity with non-computerized measures (Abend, Dan, Maoz, Raz, \& Bar-Haim, 2014).

Anxiety and depressive symptoms. The Hospital Anxiety and Depression Scale (HADS) was used to measure anxiety and depression (Zigmond \& Snaith, 1983). The HADS is a 14-item self-report questionnaire with a 7-item anxiety subscale and 7-item depression subscale. Each subscale can be used independently, and scores range from 0 to 21 for each subscale; a total score out of 42 is calculated, with higher scores indicating greater levels of anxiety, depression and overall psychological distress. The HADS is responsive to change in longitudinal trials of those with COPD and demonstrates responsiveness of Cohen's D from -0.45 to -0.62 , and a minimal clinically important difference of 1.6 (Smid et al., 2017). Subscale scores of eight or higher indicate probable anxiety and/or depressive symptoms; the HADS has well established validity, reliability, and diagnostic accuracy for measurement of anxiety and depressive symptom in patients with COPD (Phan et al., 2016).

\section{Procedure}

Ethical considerations. The study site's Institutional Review Board approved this pilot study prior to participant recruitment (approval number: BHL-17-1435). All participants gave written informed consent to participate in this study. Data were collected from December 2017 to February 2018.

Recruitment and enrollment. Before enrollment, potential participants were screened for cognitive impairment using the MiniCog (Borson et al., 2003) and for adequate health literacy using the Newest Vital Sign (Weiss et al., 2005). Confirmation of home Wi-Fi and a smart phone with text-messaging capabilities were obtained. After determination of adequate health literacy and absence of obvious cognitive impairment, potential participants gave written informed consent and were provided with a tablet-computer. Extensive training on use of the tablet was then administered. Each participant was also given written instructions and reminders use of the tablet and how to access the intervention. After orientation to the tablet, baseline data collection took place in an empty exam room. All measures were completed and automatically transmitted via W-Fi to an encrypted data collection system (Research Electronic Data Capture), located behind a University firewall.

For the first week (days 1-7) patients were given access to a restricted version of the website to acclimate to navigating the tablet and the study's website - this period provided standard medical education provided by the recruiting clinic. Troubleshooting and issues with accession were addressed during this time period. On Day 8, participants were granted access to the whole website and intervention package. Given this was the initial testing of this intervention, the participants were instructed to use the modules in any way they chose so that we could observe their natural interactions with the intervention and platform.

Text messaging to promote adherence. Participants received daily text messages between 1600 and 1900 hours on Days 2 to 21 to remind them to acclimate to the website, explore the educational modules, and complete the respective measures when indicated.

\section{Data analysis}

Descriptive statistics including means (standard deviations) and frequencies were used to characterize the sample and the variables studies. Three repeated measures ANOVAs (RM-ANOVA) were conducted to compare mean scores for a perceived self-care ability, anxiety, and depressive symptoms at baseline to measures collected on day 8 , day 15 , and day 21. Paired sample t-tests were conducted to compare mean perceived self-care adherence, self-care information needs scores, and somatic symptom scores between pre- and post intervention. Spearman correlation coefficients were 


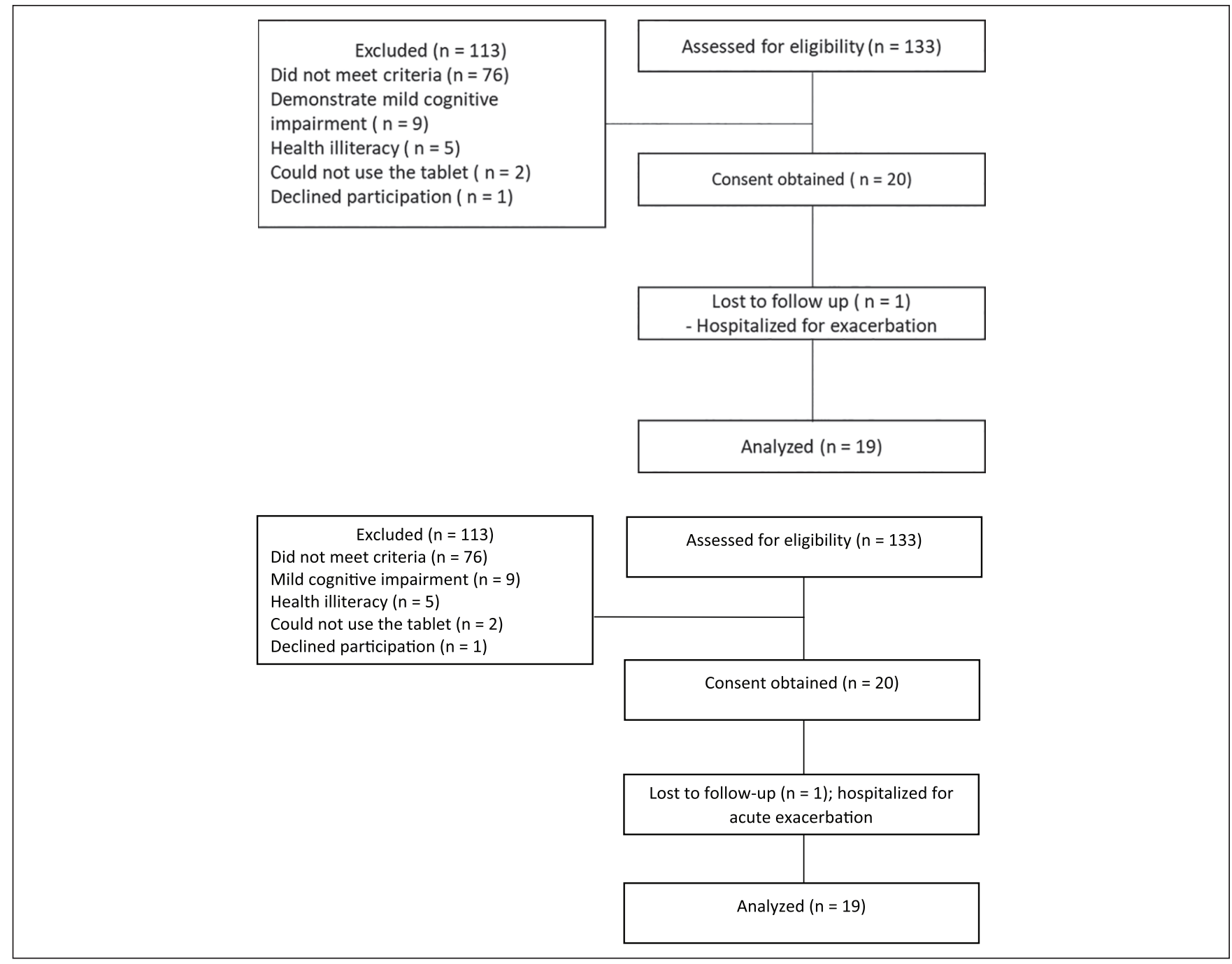

Figure I. Screening and Enrollment Flow Diagram.

used to evaluate the associations among variables based on the ordinal level of the data. Website usability was summarized using descriptive statistics. Although it was not the intent to investigate for significant differences of our variables over time, analyses were performed using SPSS version 24 (IBM, Armonk, NY) with an a priori $\alpha=0.05$ to indicate significance-we did not adjust our significance level to account for multiple comparisons of outcome variables due to the exploratory nature of the data analysis.

\section{Digital Intervention Usability}

Usability of the intervention is described by the total number of time the website was accessed, time spent on the website, medium used to access the website, and adherence to weekly measures. General acceptability was also described. Participant's usage of the tablet and webpage were tracked using Google Analytic Software.

\section{Results}

A total of 133 patients were screened for eligibility (Figure 1.). Thirty-seven met eligibility criteria and 20 (54\%) patients were able and willing to participate. Seventeen were excluded; nine for obvious cognitive impairment, five for inadequate health literacy, two due to an inability to use the tablet, and one refused enrollment despite meeting all inclusion and exclusion criteria. Participant recruitment and follow-up took place from December 2017 to February 2018.

\section{Characteristics of the Participants}

Participants $(\mathrm{N}=20)$ were primarily female, obese, Caucasian, with an averaged 62 years of age (Tables 2 and 3). A majority of participants had at least a high school education, were single, widowed, or divorced, and were not working due to disability, retirement or lack of employment. A majority of 
Table 2. Characteristics of Participants $(N=20)$.

\begin{tabular}{lr}
\hline Characteristics & $n(\%)$ \\
\hline Female & $13(65 \%)$ \\
Ethnicity & \\
$\quad$ Caucasian & $18(90 \%)$ \\
Highest Education Level & \\
$\quad$ Less than high school & $8(40 \%)$ \\
High school graduate or above & $12(60 \%)$ \\
Marital Status & \\
Single & $8(40 \%)$ \\
Married & $7(35 \%)$ \\
Widowed/divorced & $5(25 \%)$ \\
Smoking Status & \\
Current smoker & $8(40 \%)$ \\
Quit smoking & $10(50 \%)$ \\
Never smoker & $2(10 \%)$ \\
Employment Status & \\
Employed & $8(40 \%)$ \\
Disabled/sick leave & $6(30 \%)$ \\
Retired/unemployed & $6(30 \%)$ \\
Live Alone & $6(30 \%)$ \\
GOLD Stage & \\
II & $9(45 \%)$ \\
III & $5(25 \%)$ \\
IV & $6(30 \%)$ \\
\hline
\end{tabular}

Abbreviations: GOLD, Global Initiative for Obstructive Lung Disease Stage.

Table 3. Characteristics of Participants $(N=20)$.

\begin{tabular}{lcc}
\hline Characteristics & Mean & Standard Deviation \\
\hline BMI in $\mathrm{kg} / \mathrm{m}^{2}$ & 30.2 & 7.6 \\
Number of comorbidities & 6.6 & 4.6 \\
$\begin{array}{l}\text { Number of exacerbations } \\
\text { in previous year }\end{array}$ & 1.5 & 1.2 \\
Number of medications & 12.9 & 6.6 \\
$\quad$ prescribed & & \\
FEV, in liters per second & 1.17 & .43 \\
\% predicted FEV, & 43 & 14.9 \\
FVC in liters & 2.5 & .73 \\
FEV,/FVC & 48 & 13.4 \\
\hline
\end{tabular}

Abbreviations: BMI, body mass index; FEV forced expiratory volume in one second; FVC, forced vital capacity.

the participants had advanced COPD (GOLD spirometric grade III or IV) and averaged 1.5 exacerbations in the previous year. Eight participants (40\%) reported they were current smokers, while $50 \%$ of participants had quit smoking at some point. Participants had over six comorbidities on average and reported being prescribed a large number of medications. Two participants had previously participated in a PR program and no participants were part of a palliative care program. It should be noted that 12 participants reported having a caregiver of some sort (formal or informal).

\section{Exploratory Analysis of Outcomes}

Comparison of perceived self-care ability, anxiety, and depressive symptoms. Perceived self-care ability, anxiety, and depressive symptom scores were measured four times (baseline, day 8, 15, and 21) and compared using a RM-ANOVA (Table 4). At baseline, anxiety scores averaged $6.6 \pm 3.3$, depressive symptoms $5.6 \pm 3.5$, and self-care ability averaged $58.9 \pm 12$. There were no significant differences in scores among the measurement times for self-care ability $[F(3,48)$ $=2.46, \mathrm{p}=.07]$, anxiety symptoms $[F(3,51)=.585, \mathrm{p}=.63]$ or depressive symptoms $[F(3,51)=0.45, \mathrm{p}=.72]$.

Comparison of self-care adherence, self-care information needs and somatic symptoms. Perceived self-care adherence, selfcare information needs, and somatic symptoms were measured at baseline and on day 21, after completion of the intervention. Baseline mean scores were compared to scores obtained on day 21 with paired samples t-tests. There was a significant improvement in adherence scores from baseline $(58.1 \pm 19.3)$ to post-intervention $(67.6 \pm 12.2) ; t(17)=$ $-2.47, \mathrm{p}=.025$. Self-care information needs were significantly reduced after the intervention (baseline: $13.7 \pm 3.1$; post intervention: $11.3 \pm 1.8 ; t(17)=2.82, \mathrm{p}=.012$ ). This indicates that adherence improved and information needs decreased, which is indicative of an increase in knowledge. There were no significant changes in any of the six measured somatic symptoms from baseline to the end of the intervention. Visual trends in the data suggest that symptoms generally were perceived as more severe at Day 21 than at baseline, however a larger study with more participants is needed to confirm this trend.

Association between perceived self-care ability, adherence, knowledge, anxiety/ depressive symptoms. The association between self-care ability and adherence was significant at the conclusion of the study $(\mathrm{p}=.016)$. There was a positive relationship $(\mathrm{r}=.56 ; 95 \%$ bias corrected confidence interval $[\mathrm{BCa} \mathrm{CI}]=.17-.81)$; as participants reported higher degrees of self-care ability, they also reported higher degrees of self-care adherence. There were no significant associations between self-care ability and knowledge in this sample, $\mathrm{r}=-.193, \mathrm{p}=.44,[.23,-.59]$. There were no significant associations between self-care ability and anxiety symptoms in this sample, $\mathrm{r}=-.157, \mathrm{p}=.54,[-.64, .42]$. Perceived selfcare ability was significantly related to depressive symptoms, $r=-.63, p<.01,[.88,-.17]$. There was an inverse relationship, meaning that as self-care ability scores increases, depressive symptoms decreased.

Website feasibility and usability. Due to technical issues regarding the website tracking software, usability statistics were only available for seven participants; two participants' data were incomplete (the analytic software was enabled for their days 11-21) and five participants' usability data were complete (the analytic software captured their usability for 
Table 4. Comparison of Mean Scores for Symptoms, Anxiety, Depressive Symptoms, Perceived Self-Care Ability, Adherence and Information Needs $(n=19)$.

\begin{tabular}{|c|c|c|c|c|c|c|c|c|c|}
\hline \multirow[b]{2}{*}{ Variable } & \multicolumn{2}{|c|}{ Baseline } & \multicolumn{2}{|c|}{ Day 8} & \multicolumn{2}{|c|}{ Day 15} & \multicolumn{2}{|c|}{ Day 21} & \multirow[b]{2}{*}{ P Value } \\
\hline & $M$ & $S D$ & M & $S D$ & M & $S D$ & M & $S D$ & \\
\hline HADS - anxiety & 6.6 & 3.3 & 6.8 & 3.9 & 6.2 & 4.1 & 6.3 & 4.3 & .63 \\
\hline HADS - depression & 5.6 & 3.5 & 5.8 & 3.1 & 5.5 & 3.3 & 6.1 & 3.4 & .72 \\
\hline Perceived self-care Ability & 58.9 & 12 & 55.5 & 13.5 & 54.3 & 14.5 & 57.4 & 14.5 & .07 \\
\hline Self-care adherence & 58.1 & 19.3 & - & - & - & - & 67.6 & 12.2 & $.03^{*}$ \\
\hline Self-care information needs & 13.7 & 3.1 & - & - & - & - & 11.3 & 1.8 & $.01 *$ \\
\hline Distress due to cough & 26.6 & 25.4 & - & - & - & - & 27.8 & 26.2 & .59 \\
\hline Distress due to mucous & 27.9 & 22.3 & - & - & - & - & 29.3 & 23.3 & .68 \\
\hline Chest tightness & 28.8 & 24.1 & - & - & - & - & 28.1 & 24.4 & .79 \\
\hline Dyspnea with activity & 45.4 & 27.8 & - & - & - & - & 48.0 & 28.9 & .27 \\
\hline Dyspnea with rest & 25.9 & 26.1 & - & - & - & - & 27.4 & 24.3 & .48 \\
\hline Fatigue & 38.0 & 26.8 & - & - & - & - & 44.8 & 27.2 & .08 \\
\hline
\end{tabular}

Note. Values are mean \pm SD.

Comparisons were performed with paired t-tests, or repeated measures analysis of variance, based on the number of repeated measures; $*$ indicates significant finding.

Abbreviations: HADS, Hospital Anxiety and Depression Scale; SMAS-18, Self-management Ability Scale - I8-item version.

days 1-21). However, in regards to instrument completion, 229 out of $240(95.4 \%)$ of all instruments, among all time points, were submitted with complete data - this indicates that the timing and structure of data collection were acceptable.

From the seven patients' website usage, there were a total of 1,120 page-views. Given that this reflects less than half of our participants, we anticipate that interaction with the intervention was substantially higher than what is reflected. The website was accessed $75.3 \%$ of the time using tablets, $20.2 \%$ using desktop computers, and $4.5 \%$ using mobile phones. The highest volume of users was recorded between $8 \mathrm{Am}$ and $10 \mathrm{AM}$, as well as between 3 PM and 8 PM on any given day; this is believed to reflect the daily variation in disease burden from morning to evening. Participants spent 6 minutes and 19 seconds per session on average, and accessed 5.66 different pages per session-given that each component of the intervention contained numerous brief subsections, we believe 5.66 page-views reflects a high degree of usage per day. The most accessed educational module was the diet/ nutritional webpage followed by the breathing control, medication, physical activity, environment modification, exacerbation, and mental health webpages, respectively. Participants overall viewed the intervention as helpful and acceptable, which feedback such as, "I would have never known some of these things about my breathing" and "This provided me with information my doctor never told me." Several participants reported that they would like to have an integration between the intervention and their primary care. Of note, four participants voiced that they would be interested in a program like this permanently, and specifically "I thought the symptom diary was helpful for me to track how I have been feeling."

\section{Discussion}

We tested the use of a digital educational intervention for patients with COPD and evaluated its effect on perceived self-care ability, self-care adherence, self-care knowledge, anxiety, and depressive symptoms. Although a small pilot study, these preliminary results are promising for future examination of this self-care intervention. Given this was the initial testing of this intervention, and was delivered at a low dose, we stimulated change in two of our outcome variables. This fundamentally suggests that adherence and knowledge can be improved with minimal intervention from the practitioner, and these variables may be able to be altered rather passively. Outcomes such as self-care ability, anxiety, and depression may require a more targeted, involved approach to elicit responses.

Results from this pilot study will likely result in moderate to substantive changes and adaptions for future testing. An important and possibly confounding finding was related to our sample. We likely had an atypical sample, similar to that reported from several of Nguyen and colleagues studies (Nguyen et al., 2013, 2015). These peoples were moderate to highly educated, were insured, had previously demonstrated self-management skills with some degree of proficiency, and were technologically savvy, which could result in limited opportunities for improvement from baseline. However, our sample performed similarly to those with COPD who participated in digital self-management interventions, who reported high degrees of comfort in using digital health tools.

In the COPD literature, people demonstrate significantly worse scores of self-care ability compared to other chronic diseases (Cramm \& Nieboer, 2012). However, the participants in this sample reported a moderate to high level of selfcare ability. This is likely multifactorial given the participants 
were a convenience sample of patients from a local pulmonary clinic and those who volunteered for participation in this study were already engaged in self-care activities to some degree, and $10 \%$ of our sample had previously participated in a PR program. A majority of our participants completed more than a high school education and we screened for adequate health literacy and satisfactory cognitive function; thus, our participants may not represent the typical patient with COPD.

The lack of change in self-care ability could be due to the short time period of the study or a lack of sensitivity of the SMAS-S to small change. Self-management abilities comprise a set of skills that are meant to be practiced, used and perfected over time; thus, self-care ability might continue to improve over time and not be reflected in these initial scores. A number of prior studies measured indices of self-care ability such as keeping follow up appointments, completing daily symptom diaries, taking prescribed medications, monitoring for changes in symptoms, and contacting providers for suspected exacerbations (Bischoff et al., 2011; Blackstock, ZuWallack, Nici, \& Lareau, 2016; Fan et al., 2012). Investigators for these studies reported no change or a gradual decline of patient adherence and skills in these behaviors (Bischoff et al., 2011; Blackstock et al., 2016; Fan et al., 2012). These declines in self-care behaviors were postulated to be related to loss of interest in self-care, increased complexity in prescribed treatment regimen, and poor quality of care from providers. However, previous investigators implemented a high dose of their intervention through repeated, structured interventions over the course of days to weeks, while we structured our intervention to be less structured and a smaller dose. Thus, it is likely that our short timeframe and low dose were not adequate to observe change. Furthermore, perceived self-care ability was already moderately high in our participants, hence it is likely the intervention did not provide an adequate dose to produce an improvement in selfcare ability.

Participants demonstrated significant improvement in perceived self-care adherence and self-care knowledge. On average, our participants improved their self-care adherence by $9.5 \%$ and reduced their self-care information needs by $9.6 \%$ after the two-week intervention period. This is consistent with previous investigators who examined the impact of self-care interventions on adherence and self-care knowledge (Leiva-Fernandez, Leiva-Fernandez, Garcia-Ruiz, Prados-Torres, \& Barnestein-Fonseca, 2014; Smit, de Vries, \& Hoving, 2012). Previous investigators tested multidimensional self-care interventions and demonstrated that the experimental group improved perceived adherence by $30.5 \%$ to $99 \%$ compared to control during longitudinal follow-up (Leiva-Fernandez et al., 2014; Smit et al., 2012). There is extensive evidence that supports various types of self-care interventions can improve COPD specific knowledge, thus, these findings are expected. Our results combined with previous findings support the need to incorporate these concepts into future self-care interventions as a means to improve adherence and self-care knowledge. However, future studies are needed to examine the long-term impact of interventions delivered using digital platforms on constructs such as selfcare adherence and knowledge in patients with COPD.

On average, our participants did not meet the HADS cut points for presence of anxiety and depressive symptoms, and there were no significant changes in anxiety and depressive symptom scores throughout the study. A number of previous investigators found self-care interventions had no effect on anxiety and depression (Effing, Zielhuis, Kerstjens, van der Valk, \& van der Palen, 2011; Panagioti, Scott, Blakemore, \& Coventry, 2014); while more recently in meta analyses of data, the investigators reported improvement in anxiety and depression scores after an intervention (Jolly et al., 2018; Usmani et al., 2017). It seems self-care interventions can improve anxiety and depression scores if participants exhibit anxiety or depressive symptoms pre-intervention. Given our participants did not report a significant degree of anxiety and depressive symptoms at baseline, it is not surprising we did not observe an improvement in HADS scores. Clearly, the initial degree of mental distress will influence the degree of change.

There are several limitations of this study. The sample for this study was small and short in duration, thus limiting our ability to detect differences for some of our analyses - this clearly warrants a longer follow-up time. Moreover, our selected instruments (self-care ability, adherence, and knowledge) have no supporting literature regarding responsiveness in longitudinal studies in COPD, thus is another limitation in our ability to detect change not due to chance in the timeframe studied. This was also a convenience sample who were relatively well educated, with adequate health literacy and normal cognitive function - thus, this sample may not be indicative of the population. The instrument used in this study was self-report, which introduced the potential for social desirability and response bias. However, the variables we measured were subjective and self-report was the only appropriate way to measure them. Use of the MOS-SAS should undergo more psychometric testing in COPD populations to ensure its usefulness in this population. Lastly, due to coding error, we lost the usability statistics from the intervention, thus limiting our ability to speculate on associations between our intervention and included intervention behaviors.

\section{Future Research}

Further testing of this intervention is necessary to further elucidate the efficacy of self-care interventions in people with COPD. Given the emphasis on both the intervention and the repeated text messages to participants, it is impossible to know which aspects had a greater effect, if any, on our observed outcomes. A sequential, multiple assignment, randomized trial (SMART) may be a future step, which would 
enable us to determine which components need to be emphasized to determine the greatest effect for people with COPD. Moreover, a second larger study should be conducted where the digital coding is correct and in place. This would enable us to draw inferences from the usability statistics of the intervention, content accessed on the Website, and outcomes measured.

Our theory-based, self-care educational intervention delivery via electronic means produced change in perceived self-care by nearly $10 \%$ and reduced knowledge needs by almost $10 \%$. The participants were highly adherent and interactive with the intervention tested in this pilot study.

\section{Declaration of Conflicting Interests}

The author(s) declared no potential conflicts of interest with respect to the research, authorship, and/or publication of this article.

\section{Funding}

The author(s) received no financial support for the research, authorship, and/or publication of this article.

\section{ORCID iD}

Andrew Bugajski iD https://orcid.org/0000-0001-8065-7288

\section{References}

Abend, R., Dan, O., Maoz, K., Raz, S., \& Bar-Haim, Y. (2014). Reliability, validity and sensitivity of a computerized visual analog scale measuring state anxiety. Journal of Behavior Therapy and Experimental Psychiatry, 45(4), 447-453. doi:10.1016/j.jbtep.2014.06.004

Bischoff, E. W., Hamd, D. H., Sedeno, M., Benedetti, A., Schermer, T. R., Bernard, S., . . Bourbeau, J. (2011). Effects of written action plan adherence on COPD exacerbation recovery. Thorax, 66(1), 26-31. doi:10.1136/thx.2009.127621

Blackstock, F. C., ZuWallack, R., Nici, L., \& Lareau, S. C. (2016). Why don't our patients with chronic obstructive pulmonary disease listen to us? The enigma of nonadherence. Annals of American Thoracic Society, 13(3), 317-323. doi:10.1513/ AnnalsATS.201509-600PS

Borson, S., Scanlan, J. M., Chen, P., \& Ganguli, M. (2003). The Mini-Cog as a screen for dementia: Validation in a populationbased sample. Journal of the American Geriatrics Society, 51(10), 1451-1454.

Cannon, D., Buys, N., Sriram, K. B., Sharma, S., Morris, N., \& Sun, J. (2016). The effects of chronic obstructive pulmonary disease self-management interventions on improvement of quality of life in COPD patients: A meta-analysis. Respiratory Medicine, 121, 81-90. doi:10.1016/j.rmed.2016.11.005

Clari, M., Matarese, M., Ivziku, D., \& De Marinis, M. G. (2017). Self-care of people with chronic obstructive pulmonary disease: A meta-synthesis. The Patient, 10(4), 407-427. doi:10.1007/ s40271-017-0218-z

Cramm, J. M., \& Nieboer, A. P. (2012). Self-management abilities, physical health and depressive symptoms among patients with cardiovascular diseases, chronic obstructive pulmonary disease, and diabetes. Patient Education and Counseling, 87(3), 411415. doi:10.1016/j.pec.2011.12.006

Cramm, J. M., \& Nieboer, A. P. (2013). The relationship between self-management abilities, quality of chronic care delivery, and wellbeing among patients with chronic obstructive pulmonary disease in The Netherlands. International Journal of Chronic Obstructive Pulmonary Disease, 8, 209-214. doi:10.2147/ copd.s42667

Cramm, J. M., Strating, M. M., de Vreede, P. L., Steverink, N., $\&$ Nieboer, A. P. (2012). Validation of the self-management ability scale (SMAS) and development and validation of a shorter scale (SMAS-S) among older patients shortly after hospitalisation. Health and Quality of Life Outcomes, 10, 9. doi:10.1186/1477-7525-10-9

Effing, T. W., Vercoulen, J. H., Bourbeau, J., Trappenburg, J., Lenferink, A., Cafarella, P., ... van der Palen, J. (2016). Definition of a COPD self-management intervention: International Expert Group consensus. European Respiratory Journal, 48(1), 46-54. doi:10.1183/13993003.00025-2016

Effing, T., Zielhuis, G., Kerstjens, H., van der Valk, P., \& van der Palen, J. (2011). Community based physiotherapeutic exercise in COPD self-management: A randomised controlled trial. Respiratory Medicine, 105(3), 418-426. doi:10.1016/j. rmed.2010.09.017

Fan, V. S., Gaziano, J. M., Lew, R., Bourbeau, J., Adams, S. G., Leatherman, S., . . . Niewoehner, D. E. (2012). A comprehensive care management program to prevent chronic obstructive pulmonary disease hospitalizations: A randomized, controlled trial. Annals of Internal Medicine, 156(10), 673-683. doi:10.7326 /0003-4819-156-10-201205150-00003

Hyland, M. E., Jones, R. C., \& Hanney, K. E. (2006). The lung information needs questionnaire: Development, preliminary validation and findings. Respiratory Medicine, 100(10), 1807-1816. doi:10.1016/j.rmed.2006.01.018

Jolly, K., Majothi, S., Sitch, A. J., Heneghan, N. R., Riley, R. D., Moore, D. J., . . J Jordan, R. E. (2016). Self-management of health care behaviors for COPD: A systematic review and meta-analysis. International Journal of Chronic Obstructive Pulmonary Disease, 11, 305-326. doi:10.2147/copd.s90812

Jolly, K., Sidhu, M. S., Bates, E., Majothi, S., Sitch, A., Bayliss, S., . . . Jordan, R. E. (2018). Systematic review of the effectiveness of community-based self-management interventions among primary care COPD patients. NPJ Primary Care Respiratory Medicine, 28(1), 44. doi:10.1038/s41533-018-0111-9

Leiva-Fernandez, J., Leiva-Fernandez, F., Garcia-Ruiz, A., PradosTorres, D., \& Barnestein-Fonseca, P. (2014). Efficacy of a multifactorial intervention on therapeutic adherence in patients with chronic obstructive pulmonary disease (COPD): A randomized controlled trial. BMC Pulmonary Medicine, 14, 70. doi:10.1186/1471-2466-14-70

Lenferink, A., Brusse-Keizer, M., van der Valk, P. D., Frith, P. A., Zwerink, M., Monninkhof, E. M., ... Effing, T. W. (2017). Self-management interventions including action plans for exacerbations versus usual care in patients with chronic obstructive pulmonary disease. Cochrane Database of Systematic Reviews, 8, CD011682. doi:10.1002/14651858.CD011682.pub2

Noar, S., \& Harrington, N. (2012). eHealth applications: Promising strategies for behavior change. Routledge. 
Nguyen, H. Q., Donesky, D., Reinke, L. F., Wolpin, S., Chyall, L., Benditt, J. O., . . . Carrieri- Kohlman, V. (2013). Internetbased dyspnea self-management support for patients with chronic obstructive pulmonary disease. Journal of Pain and Symptom Management, 46(1), 43-55. doi:10.1016/j.jpainsymman.2012.06.015

Nguyen, H. Q., Gill, D. P., Wolpin, S., Steele, B. G., \& Benditt, J. O. (2009). Pilot study of a cell phone-based exercise persistence intervention post-rehabilitation for COPD. International Journal of Chronic Obstructive Pulmonary Disease, 4, 301-313.

Nguyen, H. Q., Harrington, A., Liu, I. L., Lee, J. S., \& Gould, M. K. (2015). Impact of pulmonary rehabilitation on hospitalizations for chronic obstructive pulmonary disease among members of an integrated health care system. Journal of Cardiopulmonary Rehabilitatoin and Prevention, 35(5), 356-366. doi:10.1097/ hcr.0000000000000128

Panagioti, M., Scott, C., Blakemore, A., \& Coventry, P. A. (2014). Overview of the prevalence, impact, and management of depression and anxiety in chronic obstructive pulmonary disease. International Journal of Chronic Obstructive Pulmonary Disease, 9, 1289-1306. doi:10.2147/copd.s72073

Phan, T., Carter, O., Adams, C., Waterer, G., Chung, L. P., Hawkins, M., . . . Strobel, N. (2016). Discriminant validity of the hospital anxiety and depression scale, Beck depression inventory (II) and Beck anxiety inventory to confirmed clinical diagnosis of depression and anxiety in patients with chronic obstructive pulmonary disease. Chronic Respiratory Disease, 13(3), 220-228. doi:10.1177/1479972316634604

Riegel, B., Jaarsma, T., Lee, C. S., \& Stromberg, A. (2018). Integrating symptoms into the middle-range theory of self-care of chronic illness. Advances in Nursing Science. doi:10.1097/ ans.0000000000000237

Riegel, B., Jaarsma, T., \& Strömberg, A. (2012). A middle-range theory of self-care of chronic illness. Advances in Nursing Science, 35(3), 194-204. 10.1097/ANS.0b013e318261b1ba

Sherbourne, C. D., Hays, R. D., Ordway, L., DiMatteo, M. R., \& Kravitz, R. L. (1992). Antecedents of adherence to medical recommendations: Results from the Medical Outcomes Study. Journal of Behavioral Medicine, 15(5), 447-468.

Smid, D. E., Franssen, F. M., Houben-Wilke, S., Vanfleteren, L. E., Janssen, D. J., Wouters, E. F., \& Spruit, M. A. (2017). Responsiveness and MCID Estimates for CAT, CCQ, and
HADS in patients with COPD undergoing pulmonary rehabilitation: A prospective analysis. Journal of the American Medical Directors Association, 18(1), 53-58. doi:10.1016/j. jamda.2016.08.002

Smit, E. S., de Vries, H., \& Hoving, C. (2012). Effectiveness of a web-based multiple tailored smoking cessation program: A randomized controlled trial among Dutch adult smokers. Journal of Medical Internet Research, 14(3), e82. doi:10.2196/ jmir. 1812

Usmani, Z. A., Carson, K. V., Heslop, K., Esterman, A. J., De Soyza, A., \& Smith, B. J. (2017). Psychological therapies for the treatment of anxiety disorders in chronic obstructive pulmonary disease. Cochrane Database Systematic Reviews, CD010673. doi:10.1002/14651858.CD010673.pub2

Webel, A. R., Frazier, S. K., Moser, D. K., \& Lennie, T. A. (2007). Daily variability in dyspnea, edema and body weight in heart failure patients. European Journal of Cardiovascular Nursing, 6(1), 60-65. doi:10.1016/j.ejcnurse.2006.04.003

Weiss, B. D., Mays, M. Z., Martz, W., Castro, K. M., DeWalt, D. A., Pignone, M. P., . . .Hale, F. A. (2005). Quick assessment of literacy in primary care: the newest vital sign. The Annals of Family Medicine, 3(6), 514-522. doi:10.1370/afm.405

World Health Organization. (2017). Chronic obstructive pulmonary disease (COPD). Retrieved from https://www.who.int/en/ news-room/fact-sheets/detail/chronic-obstructive-pulmonarydisease-(copd)

Wouters, E. F. M., Wouters, B., Augustin, I. M. L., Houben-Wilke, S., Vanfleteren, L., \& Franssen, F. M. E. (2018). Personalised pulmonary rehabilitation in COPD. European Respiratory Review, 27(147). doi:10.1183/16000617.0125-2017

Yohannes, A. M., \& Alexopoulos, G. S. (2014). Depression and anxiety in patients with COPD. The European Respiratory Review, 23(133), 345-349. doi:10.1183/09059180.00007813

Zigmond, A. S., \& Snaith, R. P. (1983). The hospital anxiety and depression scale. Acta Psychiatrica Scandinavica, 67(6), 361-370.

Zwerink, M., Brusse-Keizer, M., van der Valk, P. D., Zielhuis, G. A., Monninkhof, E. M., van der Palen, J., Frith, P. A., \& Effing, T. (2014). Self management for patients with chronic obstructive pulmonary disease. Cochrane Database of Systematic Reviews, 19(3), CD002990. 